MATHEMATICS OF COMPUTATION

Volume 67, Number 222, April 1998, Pages 457-477

S 0025-5718(98)00930-2

\title{
IMPLICIT-EXPLICIT MULTISTEP FINITE ELEMENT METHODS FOR NONLINEAR PARABOLIC PROBLEMS
}

\author{
GEORGIOS AKRIVIS, MICHEL CROUZEIX, AND CHARALAMBOS MAKRIDAKIS
}

\begin{abstract}
We approximate the solution of initial boundary value problems for nonlinear parabolic equations. In space we discretize by finite element methods. The discretization in time is based on linear multistep schemes. One part of the equation is discretized implicitly and the other explicitly. The resulting schemes are stable, consistent and very efficient, since their implementation requires at each time step the solution of a linear system with the same matrix for all time levels. We derive optimal order error estimates. The abstract results are applied to the Kuramoto-Sivashinsky and the CahnHilliard equations in one dimension, as well as to a class of reaction diffusion equations in $\mathbb{R}^{\nu}, \nu=2,3$.
\end{abstract}

\section{INTRODUCTION}

In this paper we construct and analyze implicit-explicit multistep schemes for the time discretization of a class of nonlinear parabolic problems of the form: Given $T>0$ and $u^{0} \in H$, find $u:[0, T] \rightarrow D(A)$ such that

$$
\begin{aligned}
& u^{\prime}(t)+A u(t)=B(u(t)), \quad 0<t<T, \\
& u(0)=u^{0},
\end{aligned}
$$

where $A$ is a linear, selfadjoint, positive definite operator on a Hilbert space $(H,(\cdot, \cdot))$ with domain $D(A)$ dense in $H$, and $B: D(A) \rightarrow H$ is a (possibly nonlinear) differentiable operator. Considering a finite dimensional subspace $V_{h}$ of $V, V:=D\left(A^{1 / 2}\right)$, we are led to a semidiscrete problem approximating (1.1): We seek a function $u_{h}$, $u_{h}(t) \in V_{h}$, defined by

$$
\begin{aligned}
& u_{h}^{\prime}(t)+A_{h} u_{h}(t)=B_{h}\left(u_{h}(t)\right), \quad 0<t<T, \\
& u_{h}(0)=u_{h}^{0} ;
\end{aligned}
$$

here $u_{h}^{0} \in V_{h}$ is a given approximation to $u^{0}$, and $A_{h}, B_{h}$ are appropriate operators on $V_{h}$ with $A_{h}$ linear, selfadjoint and positive definite.

Following an idea of Crouzeix, [3], for the time discretization of parabolic equations with time dependent coefficients, we combine implicit and explicit multistep schemes to discretize (1.2) in time: Implicit schemes are used for discretizing the left-hand side of the o.d.e. in (1.2), and explicit schemes for the nonlinear righthand side. Thus, letting $k$ be a (constant) time step, $t_{n}=n k, n=0, \ldots, N$,

Received by the editor July 3, 1995 and, in revised form, December 8, 1995.

1991 Mathematics Subject Classification. Primary 65M60, 65M12; Secondary 65L06.

The work of the first and third authors was supported in part by a research grant from the University of Crete. 
$T=N k$, we define a sequence of approximations $U^{n}, U^{n} \in V_{h}$, to $u^{n}=u\left(t_{n}\right)$, by

$$
\sum_{i=0}^{q} \alpha_{i} U^{n+i}+k \sum_{i=0}^{q} \beta_{i} A_{h} U^{n+i}=k \sum_{i=0}^{q-1} \gamma_{i} B_{h}\left(U^{n+i}\right) .
$$

A scheme of this form is characterized by three polynomials $\alpha, \beta$ and $\gamma$,

$$
\alpha(\zeta)=\sum_{i=0}^{q} \alpha_{i} \zeta^{i}, \quad \beta(\zeta)=\sum_{i=0}^{q} \beta_{i} \zeta^{i}, \quad \gamma(\zeta)=\sum_{i=0}^{q-1} \gamma_{i} \zeta^{i} .
$$

We note that, when $B_{h}$ vanishes, the scheme (1.3) reduces to the implicit linear multistep method $(\alpha, \beta)$ (or $(\rho, \sigma)$ in the notation of Dahlquist) for the equation $u_{h}^{\prime}(t)+A_{h} u_{h}(t)=0$; similarly if $A_{h}$ vanishes, the scheme (1.3) reduces to the linear multistep method $(\alpha, \gamma)$, which is explicit since $\gamma_{q}=0$, for the equation $u_{h}^{\prime}(t)=B_{h}\left(u_{h}(t)\right)$. The scheme (1.3) is a combination of the methods $(\alpha, \beta)$ and $(\alpha, \gamma)$; it is linearly implicit and nonlinearly explicit. We shall refer to it as the $(\alpha, \beta, \gamma)$ scheme.

We shall assume in the sequel that the method $(\alpha, \beta)$ is strongly $A(0)$-stable; this implies, in particular, that $\alpha_{q} \beta_{q}$ is positive, which in turn ensures invertibility of the operator $\alpha_{q} I+k \beta_{q} A_{h}$. Thus, given $U^{0}, \ldots, U^{q-1}$ in $V_{h}, U^{q}, \ldots, U^{N}$ are well defined by the $(\alpha, \beta, \gamma)$ scheme. We will assume in the sequel, without loss of generality, that both $\alpha_{q}$ and $\beta_{q}$ are positive.

For the analysis of the scheme (1.3) we will need some additional assumptions for the operators $A$ and $B$ as well as for the finite dimensional spaces $V_{h}$; the operators $A_{h}$ and $B_{h}$ will then be appropriately defined. Let, thus, $|\cdot|$ denote the norm of $H$, and introduce in $V$ the norm $\|\cdot\|$ by $\|v\|=\left(A^{1 / 2} v, A^{1 / 2} v\right)^{1 / 2}$. We identify $H$ with its dual, and denote by $V^{\prime}$ the dual of $V$, again by $(\cdot, \cdot)$ the duality pairing on $V^{\prime}$ and $V$, and by $\|\cdot\|_{\star}$ the dual norm on $V^{\prime}$. We assume that $B$ can be extended to an operator from $V$ into $V^{\prime}$, which is differentiable, and an estimate of the form

$$
\left|\left(B^{\prime}(v) w, \omega\right)\right| \leq \lambda\|w\|\|\omega\|+\mu(v)|w||\omega| \quad \forall v, w, \omega \in V
$$

holds with a constant $\lambda<1$ and a functional $\mu(v)$ which is bounded for $v$ bounded in $V$. Indeed, depending on the particular $(\alpha, \beta, \gamma)$ scheme, we shall need to assume that the constant $\lambda$ is appropriately small. Further, the assumption that $\mu(v)$ is bounded for $v$ bounded in $V$ will suffice to derive our results under some mild meshconditions; these conditions can be weakened if $\mu(v)$ is bounded for $v$ bounded in some appropriate weaker norms, and even avoided if $\mu(v)$ is bounded for $v$ bounded in $H$.

We will assume in the sequel that (1.1) possesses a solution which is sufficiently regular for our results to hold. Uniqueness of smooth solutions follows easily in view of (1.4).

For the space discretization we use a family $V_{h}, 0<h<1$, of finite dimensional subspaces of $V$. In the sequel the following discrete operators will play an essential role: Define $P_{o}: V^{\prime} \rightarrow V_{h}, R_{h}: V \rightarrow V_{h}, A_{h}: V \rightarrow V_{h}$, and the nonlinear operator $B_{h}: V \rightarrow V_{h}$ by

$$
\begin{aligned}
\left(P_{o} v, \chi\right) & =(v, \chi) \quad \forall \chi \in V_{h}, \\
\left(A R_{h} v, \chi\right) & =(A v, \chi) \quad \forall \chi \in V_{h}, \\
\left(A_{h} \varphi, \chi\right) & =(A \varphi, \chi) \quad \forall \chi \in V_{h}, \\
\left(B_{h}(\varphi), \chi\right) & =(B(\varphi), \chi) \quad \forall \chi \in V_{h} .
\end{aligned}
$$


Then, obviously, $A_{h} R_{h}=P_{o} A$ and $B_{h}=P_{o} B$.

In the error analysis we shall use the approximation properties of the "elliptic projection" $R_{h}$. We assume that $R_{h} v$ is an approximation to $v$ of order $r$, provided that $v$ is sufficiently regular,

$$
\left|v-R_{h} v\right|+h^{d / 2}\left\|v-R_{h} v\right\| \leq M(v) h^{r},
$$

where $r$ and $d$ are two integers, $2 \leq d \leq r$, and $M(v)$ is bounded if an appropriate norm of $v$ is bounded. We further assume that

$$
\left\|B(v)-B\left(R_{h} v\right)\right\|_{\star} \leq M(v) h^{r} .
$$

We emphasize that the condition (1.6) serves consistency purposes only. It is needed to prove consistency of the $(\alpha, \beta, \gamma)$ scheme - and, in fact, already of the semidiscrete problem (1.2) - for $R_{h} u$ of optimal order with respect to $h$. It is somehow restrictive though, since it essentially means that, if $A$ is a differential operator of order $d$, then $B$ may contain derivatives of up to order $d / 2$ only. For some concrete differential equations, however, one can get by with a less stringent condition by taking into account in the definition of $R_{h}$ the terms of $B$ of order higher than $d / 2$.

The scheme (1.3) is very efficient since its implementation requires at every time step solving a linear system with the same matrix for all time levels. If the order of both the implicit and the explicit scheme is $p$, then under some mild meshconditions and for appropriately small $\lambda$, and appropriate starting values $U^{0}, \ldots, U^{q-1}$, we derive the optimal order error estimate

$$
\max _{0 \leq n \leq N}\left|u\left(t_{n}\right)-U^{n}\right| \leq c\left(k^{p}+h^{r}\right) .
$$

An outline of the paper is as follows: Section 2 is devoted to the analysis of a simple one step semiexplicit scheme of first order accuracy; its purpose is to motivate the analysis, in section 3, of more general multistep schemes of higher accuracy. Finally, in the last section, we apply our abstract results to three examples, namely the Kuramoto-Sivashinsky equation and the Cahn-Hilliard equation in one space dimension, and to a class of reaction diffusion equations in $\mathbb{R}^{\nu}, \nu=2,3$.

\section{AN IMPLICIT-EXPLICIT ONE STEP SCHEME}

As a motivation for the analysis of multistep schemes, we study in this section the simplest implicit-explicit scheme which is a combination of the backward Euler and the Euler method.

Let $U^{0} \in V_{h}$ be given. We define fully discrete approximations $U^{n} \in V_{h}$ to $u\left(t_{n}\right)$, recursively by

$$
\frac{U^{n+1}-U^{n}}{k}+A_{h} U^{n+1}=B_{h}\left(U^{n}\right), \quad n=0, \ldots, N-1 .
$$

Our main concern in this section is to analyze the approximation properties of the sequence $\left\{U^{n}\right\}$. As an intermediate step, we shall show consistency of the scheme (2.1) for the elliptic projection of the solution $u$ of (1.1). Let

$$
W(t)=R_{h} u(t), \quad W(t) \in V_{h}, \quad 0 \leq t \leq T .
$$


We note for later use that, in view of the definition of $R_{h},\|W(t)\|$ is obviously bounded by $\|u(t)\|$, and thus bounded uniformly in $h$ and $t$,

$$
\sup _{h} \sup _{t}\|W(t)\| \leq C \text {. }
$$

Consistency. The consistency error $E^{n}$ of the scheme (2.1) for $W$ is given by

$$
k E^{n}=\left(W^{n+1}-W^{n}\right)+k A_{h} W^{n+1}-k B_{h}\left(W^{n}\right), \quad n=0,1, \ldots, N-1 .
$$

Using the relation $A_{h} R_{h}=P_{o} A$ and the definition of $B_{h}$, we rewrite $E^{n}$ as

$$
k E^{n}=R_{h}\left(u^{n+1}-u^{n}\right)+k P_{o} A u^{n+1}-k P_{o} B\left(R_{h} u^{n}\right),
$$

and thus, in view of (1.1), $E^{n}=E_{1}^{n}+E_{2}^{n}$, where

$$
\begin{aligned}
k E_{1}^{n}= & \left(R_{h}-P_{o}\right)\left(u^{n+1}-u^{n}\right)+P_{o}\left(u^{n+1}-u^{n}-k u^{\prime}\left(t_{n+1}\right)\right) \\
& +k P_{o}\left(B\left(u^{n+1}\right)-B\left(u^{n}\right)\right),
\end{aligned}
$$

and

$$
E_{2}^{n}=P_{o}\left(B\left(u^{n}\right)-B\left(R_{h} u^{n}\right)\right) .
$$

Obviously

$$
\max _{0 \leq n \leq N-1}\left|E_{1}^{n}\right| \leq C\left(k+h^{r}\right) .
$$

Further, in view of (1.6),

$$
\max _{0 \leq n \leq N-1}\left\|E_{2}^{n}\right\|_{\star} \leq C h^{r} .
$$

Next, we show optimal order rate of convergence of our approximations to the (sufficiently regular) solution of (1.1), provided that the initial approximation $U^{0} \in$ $V_{h}$ satisfies

$$
\left|u^{0}-U^{0}\right|+h^{d / 2}\left\|u^{0}-U^{0}\right\| \leq c(u) h^{r} .
$$

We first introduce some more notation: For $v \in V$ and $b>0$, let

$$
\|v\|_{b}:=\left\{|v|^{2}+b k\|v\|^{2}\right\}^{1 / 2}
$$

and set $\|v\|:=\|v\|_{1}$. Further, let

$$
m:=\sup \left\{\mu(v):\|v\| \leq \sup _{t}\|u(t)\|+1\right\}
$$

with $\mu(v)$ as in (1.4).

The main result in this section is given in the following theorem:

Theorem 2.1. Assume that $k$ and $h^{2 r} k^{-1}$ are sufficiently small. Then, we have the local stability estimate

$$
\begin{aligned}
\left\|W^{n}-U^{n}\right\|_{\lambda} \leq e^{m t_{n}}\left\{\left\|W^{0}-U^{0}\right\|_{\lambda}\right. & +k \sum_{j=0}^{n-1}\left|E_{1}^{j}\right| \\
& \left.+\frac{1}{\sqrt{2(1-\lambda)}}\left(k \sum_{j=0}^{n-1}\left\|E_{2}^{j}\right\|_{\star}^{2}\right)^{1 / 2}\right\},
\end{aligned}
$$

and the error estimate

$$
\max _{0 \leq n \leq N}\left|u\left(t_{n}\right)-U^{n}\right| \leq C\left(k+h^{r}\right) .
$$


Proof. Let $\rho^{n}=u^{n}-W^{n}$ and $\vartheta^{n}=W^{n}-U^{n}, n=0, \ldots, N$. Then, according to (1.5),

$$
\max _{0 \leq n \leq N}\left|\rho^{n}\right| \leq C h^{r}
$$

Further, in view of (1.5) and (2.8),

$$
\begin{aligned}
\left\|\vartheta^{0}\right\|_{\lambda} & \leq\left|\vartheta^{0}\right|+k^{1 / 2}\left\|\vartheta^{0}\right\| \\
& \leq C h^{r}+C k^{1 / 2} h^{r-d / 2},
\end{aligned}
$$

i.e., since $d \leq r$,

$$
\left\|\vartheta^{0}\right\|_{\lambda} \leq C\left(k+h^{r}\right) .
$$

Now, if we assume that (2.10) holds, using (2.13), (2.6) and (2.7), we obtain

$$
\max _{0 \leq n \leq N}\left\|\vartheta^{n}\right\|_{\lambda} \leq C\left(k+h^{r}\right)
$$

and then (2.11) follows in view of (2.12). Thus, it remains to prove (2.10). To this end, we proceed as follows: From (2.1) and (2.3) we obtain an error equation for $\vartheta^{n}$,

$$
\begin{aligned}
\vartheta^{n+1}+k A_{h} \vartheta^{n+1} & =\vartheta^{n}+k\left(B_{h}\left(W^{n}\right)-B_{h}\left(U^{n}\right)\right)+k E^{n} \\
& =\vartheta^{n}+k \int_{0}^{1} B_{h}^{\prime}\left(W^{n}-s \vartheta^{n}\right) \vartheta^{n} d s+k E^{n}, \quad n=0, \ldots, N-1 .
\end{aligned}
$$

According to $(2.13),(2.6)$ and $(2.7)$, there exists a constant $C_{\star}$ such that

$$
\begin{aligned}
e^{m T}\left\{\left\|W^{0}-U^{0}\right\|_{\lambda}+k \sum_{j=0}^{N-1}\left|E_{1}^{j}\right|+\frac{1}{\sqrt{2(1-\lambda)}}\right. & \left.\left(k \sum_{j=0}^{N-1}\left\|E_{2}^{j}\right\|_{\star}^{2}\right)^{1 / 2}\right\} \\
& \leq C_{\star}\left(k+h^{r}\right) .
\end{aligned}
$$

Next, we split the error $\vartheta^{n}$ as $\vartheta^{n}=\vartheta_{1}^{n}+\vartheta_{2}^{n}$. Here $\vartheta_{1}^{0}=\vartheta^{0}, \vartheta_{2}^{0}=0$, and $\vartheta_{i}^{n}$, $n=1, \ldots, N$, are recursively defined by

$$
\vartheta_{i}^{n+1}+k A_{h} \vartheta_{i}^{n+1}=\vartheta_{i}^{n}+k \int_{0}^{1} B_{h}^{\prime}\left(W^{n}-s \vartheta^{n}\right) \vartheta_{i}^{n} d s+k E_{i}^{n}, \quad i=1,2 .
$$

We shall show inductively that, for $n=0, \ldots, N$,

$$
\left\|\vartheta_{1}^{n}\right\|_{\lambda} \leq e^{m t_{n}}\left\{\left\|\vartheta_{1}^{0}\right\|_{\lambda}+k \sum_{j=0}^{n-1}\left|E_{1}^{j}\right|\right\}
$$

and

$$
\left\|\vartheta_{2}^{n}\right\|_{\lambda} \leq e^{m t_{n}} \frac{1}{\sqrt{2(1-\lambda)}}\left(k \sum_{j=0}^{n-1}\left\|E_{2}^{j}\right\|_{\star}^{2}\right)^{1 / 2} .
$$

Obviously, (2.10) follows from (2.17) and (2.18). The estimates (2.17) and (2.18) are of course valid for $n=0$. Assume that they hold for some $n, 0 \leq n \leq N-1$. Then, according to (2.15) and to (2.10), which also holds for $n$, we have, for $k$ and $k^{-1} h^{2 r}$ small enough,

$$
\left\|\vartheta^{n}\right\| \leq \frac{1}{\sqrt{\lambda}} C_{\star}\left(k^{1 / 2}+k^{-1 / 2} h^{r}\right) \leq 1,
$$


i.e.,

$$
\sup _{0<s<1} \mu\left(W^{n}-s \vartheta^{n}\right) \leq m .
$$

Taking in (2.16) the inner product with $\vartheta_{i}^{n+1}$ and using the definition of $A_{h}$ and $B_{h}$, we obtain

$$
\left\|\vartheta_{i}^{n+1}\right\|^{2}=\left(\vartheta_{i}^{n}, \vartheta_{i}^{n+1}\right)+k \int_{0}^{1}\left(B_{h}^{\prime}\left(W^{n}-s \vartheta^{n}\right) \vartheta_{i}^{n}, \vartheta_{i}^{n+1}\right) d s+k\left(E_{i}^{n}, \vartheta_{i}^{n+1}\right),
$$

and thus, in view of (1.4) and (2.19),

$$
\left\|\vartheta_{i}^{n+1}\right\|^{2} \leq(1+m k)\left|\vartheta_{i}^{n}\right|\left|\vartheta_{i}^{n+1}\right|+\lambda k\left\|\vartheta_{i}^{n}\right\|\left\|\vartheta_{i}^{n+1}\right\|+k\left(E_{i}^{n}, \vartheta_{i}^{n+1}\right), i=1,2 .
$$

Therefore,

$$
\begin{aligned}
\left\|\vartheta_{1}^{n+1}\right\|_{\lambda}^{2} & \leq(1+m k)\left(\left|\vartheta_{1}^{n}\right|\left|\vartheta_{1}^{n+1}\right|+\lambda k\left\|\vartheta_{1}^{n}\right\|\left\|\vartheta_{1}^{n+1}\right\|\right)+k\left|E_{1}^{n}\right|\left|\vartheta_{1}^{n+1}\right| \\
& \leq(1+m k)\left\|\vartheta_{1}^{n}\right\|_{\lambda}\left\|\vartheta_{1}^{n+1}\right\|_{\lambda}+k\left|E_{1}^{n}\right|\left\|\vartheta_{1}^{n+1}\right\|_{\lambda}
\end{aligned}
$$

i.e.,

$$
\left\|\vartheta_{1}^{n+1}\right\|_{\lambda} \leq(1+m k)\left\|\vartheta_{1}^{n}\right\|_{\lambda}+k\left|E_{1}^{n}\right|
$$

From (2.21) and the induction hypothesis, it easily follows that (2.17) holds also for $n+1$. Similarly,

$$
\begin{aligned}
\left\|\vartheta_{2}^{n+1}\right\|^{2} \leq & \frac{1}{2}(1+m k)^{2}\left|\vartheta_{2}^{n}\right|^{2}+\frac{1}{2}\left|\vartheta_{2}^{n+1}\right|^{2}+\frac{1}{2} \lambda k\left\|\vartheta_{2}^{n}\right\|^{2}+\frac{1}{2} \lambda k\left\|\vartheta_{2}^{n+1}\right\|^{2} \\
& +\frac{1}{4(1-\lambda)} k\left\|E_{2}^{n}\right\|_{\star}^{2}+(1-\lambda) k\left\|\vartheta_{2}^{n+1}\right\|,
\end{aligned}
$$

i.e.,

$$
\left\|\vartheta_{2}^{n+1}\right\|_{\lambda}^{2} \leq(1+m k)^{2}\left|\vartheta_{2}^{n}\right|^{2}+\lambda k\left\|\vartheta_{2}^{n}\right\|^{2}+\frac{1}{2(1-\lambda)} k\left\|E_{2}^{n}\right\|_{\star}^{2},
$$

and, therefore,

$$
\left\|\vartheta_{2}^{n+1}\right\|_{\lambda}^{2} \leq(1+m k)^{2}\left\|\vartheta_{2}^{n}\right\|_{\lambda}^{2}+\frac{1}{2(1-\lambda)} k\left\|E_{2}^{n}\right\|_{\star}^{2} .
$$

From (2.22) and the induction hypothesis, it easily follows that (2.18) holds for $n+1$ as well, and the proof is complete.

Remark 2.1. The weak meshcondition " $k^{-1} h^{2 r}$ small" is used in the proof of Theorem 2.1 only to show that $\left\|\vartheta^{n}\right\| \leq 1$ which implies (2.19). If $\mu(v)$ is bounded for $v$ bounded in a weaker norm, one can get by with an even weaker meshcondition. Assume, for instance, that $\mu(v)$ is bounded for $v$ bounded in a norm $\|\cdot\|^{\star}$, for which an inequality of the form

$$
\|v\|^{\star} \leq|v|+|v|^{1-a}\|v\|^{a}, \quad v \in V,
$$

holds for some constant $a, 0 \leq a<1$. Then, assuming that (2.17) and (2.18) hold for some $n, 0 \leq n \leq N-1$, according to (2.15) and (2.10), which is also valid for $n$, we have

$$
\begin{aligned}
\left\|\vartheta^{n}\right\|^{\star} & \leq\left|\vartheta^{n}\right|+\left|\vartheta^{n}\right|^{1-a}\left\|\vartheta^{n}\right\|^{a} \\
& \leq C_{\star}\left(k+h^{r}\right)+C_{\star} \lambda^{-a / 2} k^{-a / 2}\left(k+h^{r}\right),
\end{aligned}
$$

and thus a condition of the form " $k$ and $k^{-a} h^{2 r}$ sufficiently small" guarantees that $\left\|\vartheta^{n}\right\|^{\star}$ is small, bounded by 1 say, and this in turn implies that (2.19) is satisfied 
for an appropriately defined constant $m$. In particular, if $\mu(v)$ is bounded for $v$ bounded in $H$, no meshcondition is required for the results of Theorem 2.1 to hold; we only have to assume that $k$ and $h$ are sufficiently small. These remarks apply equally well to the more general multistep schemes which will be investigated in the next section.

In the applications in Section 4, in the case of the Kuramoto-Sivashinsky equation $\mu(v)$ will be bounded for $v$ bounded in $H$, and no meshcondition will be needed; for the Cahn-Hilliard equation, $\mu(v)$ will be bounded for $v$ bounded in a norm $\|\cdot\|^{\star}$ for which (2.23) holds with $a=\frac{1}{2}$, and thus we only have to assume that $k$ and $k^{-1} h^{4 r}$ are sufficiently small.

Similarly, the same idea can be applied also when $\mu(v)$ is bounded for $v$ bounded in a stronger norm. Then, in special cases, the convergence result of Theorem 2.1 is still valid but under a stronger meshcondition. A particular example, where this can be done, is analyzed in section 4 .

Remark 2.2. The assumption $V_{h} \subset V$ is not essential in our analysis. One can treat the case $V_{h} \nsubseteq V$ by redefining the discrete operator $B_{h}$ and appropriately modifying the assumptions (1.4) and (1.6). We shall not dwell on this.

\section{Multistep schemes}

In this section we shall analyze implicit-explicit multistep schemes of higher order accuracy.

Let $(\alpha, \beta)$ be an implicit multistep scheme and $\alpha(\cdot), \beta(\cdot)$ be the corresponding polynomials introduced in section 1 . We order the roots $\zeta_{j}(x), 1 \leq j \leq q$ (resp. $\left.\zeta_{j}(\infty)\right)$ of the polynomial $\varpi_{x}(\cdot)=\alpha(\cdot)+x \beta(\cdot)(\operatorname{resp} . \beta(\cdot))$ in such a way that the functions $\zeta_{j}(\cdot)$ are continuous on $[0,+\infty]$ and that the roots $\xi_{j}:=\zeta_{j}(0), j=1, \ldots, s$, satisfy $\left|\xi_{j}\right|=1$; these unimodular roots are called the principal roots of $\alpha(\cdot)$ and the complex numbers $\lambda_{j}=\frac{\beta\left(\xi_{j}\right)}{\xi_{j} \alpha^{\prime}\left(\xi_{j}\right)}$ are called the growth factors of $\xi_{j}$. We assume that the method $(\alpha, \beta)$ is strongly $A(0)$-stable, that means,

$$
\text { for all } 0<x \leq \infty \text { and for all } j=1, \ldots, q \text {, there holds }\left|\zeta_{j}(x)\right|<1 \text {, }
$$

and

(ii) the principal roots of $\alpha$ are simple and satisfy: $\operatorname{Re} \lambda_{j}>0, j=1, \ldots, s$.

We also consider an explicit multistep scheme $(\alpha, \gamma)$ and we assume that both methods $(\alpha, \beta)$ and $(\alpha, \gamma)$ are of order $p$, i.e., (with the convention $0^{0}=1$ )

$$
\sum_{i=0}^{q} i^{\ell} \alpha_{i}=\ell \sum_{i=0}^{q} i^{\ell-1} \beta_{i}=\ell \sum_{i=0}^{q-1} i^{\ell-1} \gamma_{i}, \quad \ell=0,1, \ldots, p .
$$

An example of a class of $(\alpha, \beta, \gamma)$ methods satisfying the above assumptions with the order $p=q$ is given by the polynomials

$$
\alpha(\zeta)=\sum_{j=1}^{q} \frac{1}{j} \zeta^{q-j}(\zeta-1)^{j}, \quad \beta(\zeta)=\zeta^{q}, \quad \text { and } \gamma(\zeta)=\zeta^{q}-(\zeta-1)^{q} .
$$

The corresponding implicit $(\alpha, \beta)$ schemes are the well-known B.D.F. methods which are strongly $A(0)$-stable for $1 \leq q \leq 6$. 
Remark 3.1. The hypothesis (iii) may be written in the equivalent form

$$
\alpha\left(e^{x}\right)=x \beta\left(e^{x}\right)+O\left(x^{p+1}\right)=x \gamma\left(e^{x}\right)+O\left(x^{p+1}\right), \quad \text { as } x \rightarrow 0,
$$

which implies

$$
\beta(y)-\gamma(y)=O\left((y-1)^{p}\right), \quad \text { as } y \rightarrow 1 .
$$

Since $\beta-\gamma$ is a polynomial of degree $q$, we necessarily have $p \leq q$; recall that, cf. Cryer [5], the strong $A(0)$-stability of the $(\alpha, \beta)$ scheme implies also that $p \leq q$. In the same paper Cryer (see also Grigorieff and Schroll [8]) shows that for any $q$ there exists an $(\alpha, \beta) q$-step $A(0)$-stable method of order $q$. Following the proof given in Hairer and Wanner, [9, Thm. 2.2, p. 270], it can be seen that these methods can be chosen to be strongly $A(0)$-stable. On the other hand, given an $(\alpha, \beta)$ method of order $p=q$, and since the degree of $\gamma$ is $\leq q-1$, we deduce from (3.1) that the $(\alpha, \gamma)$ scheme will be of order $q$ if and only if $\gamma(\zeta)=\beta(\zeta)-\beta_{q}(\zeta-1)^{q}$.

In the sequel assume that we are given approximations $U^{0}, U^{1}, \ldots, U^{q-1} \in V_{h}$ to $u^{0}, \ldots, u^{q-1}$ such that

$$
\sum_{j=0}^{q-1}\left(\left|W^{j}-U^{j}\right|+k^{1 / 2}\left\|W^{j}-U^{j}\right\|\right) \leq c\left(k^{p}+h^{r}\right) .
$$

We define $U^{n} \in V_{h}, n=q, \ldots, N$, recursively by the $(\alpha, \beta, \gamma)$ scheme (1.3). We shall prove in this section that the method (1.3) is stable, and we shall show convergence of the approximations $U^{n}$ to $u\left(t_{n}\right)$, as $h$ and $k$ tend to zero. In particular, under a mild meshcondition and for $\lambda$ sufficiently small, we derive the optimal order error estimate

$$
\max _{0 \leq n \leq N}\left|u\left(t_{n}\right)-U^{n}\right| \leq c\left(k^{p}+h^{r}\right) .
$$

As in the previous section, we shall first show consistency of the scheme (1.3) for the projection $W, W(t)=R_{h} u(t), 0 \leq t \leq T$.

Consistency. The consistency error $E^{n}$ of the scheme (1.3) for $W$ is given by

$$
\begin{gathered}
k E^{n}=\sum_{i=0}^{q} \alpha_{i} W^{n+i}+k \sum_{i=0}^{q} \beta_{i} A_{h} W^{n+i}-k \sum_{i=0}^{q-1} \gamma_{i} B_{h}\left(W^{n+i}\right), \\
n=0, \ldots, N-q .
\end{gathered}
$$

Using the relations $A_{h} R_{h}=P_{o} A$ and $B_{h}=P_{o} B$, we can rewrite (3.3) as

$$
k E^{n}=R_{h} \sum_{i=0}^{q} \alpha_{i} u^{n+i}+k P_{o} \sum_{i=0}^{q} \beta_{i} A u^{n+i}-k P_{o} \sum_{i=0}^{q-1} \gamma_{i} B\left(R_{h} u^{n+i}\right),
$$

and thus, in view of (1.1), we split $E^{n}$ as $E^{n}=E_{1}^{n}+E_{2}^{n}$, where

$$
\begin{gathered}
k E_{1}^{n}=\left(R_{h}-P_{o}\right) \sum_{i=0}^{q} \alpha_{i} u^{n+i}+P_{o} \sum_{i=0}^{q}\left(\alpha_{i} u^{n+i}-k \beta_{i} u^{\prime}\left(t_{n+i}\right)\right) \\
+k P_{o}\left(\sum_{i=0}^{q} \beta_{i} B\left(u^{n+i}\right)-\sum_{i=0}^{q-1} \gamma_{i} B\left(u^{n+i}\right)\right)
\end{gathered}
$$


and

$$
E_{2}^{n}=P_{o} \sum_{i=0}^{q-1} \gamma_{i}\left(B\left(u^{n+i}\right)-B\left(R_{h} u^{n+i}\right)\right) .
$$

First, we will estimate $E_{1}^{n}$. Using (1.5) and the fact that $\alpha_{1}+\cdots+\alpha_{q}=0$, we easily see that

$$
\left|\left(R_{h}-P_{o}\right) \sum_{i=0}^{q} \alpha_{i} u^{n+i}\right| \leq C k h^{r} .
$$

Further, in view of the consistency properties of $(\alpha, \beta)$,

$$
\left|\sum_{i=0}^{q}\left(\alpha_{i} u^{n+i}-k \beta_{i} u^{\prime}\left(t_{n+i}\right)\right)\right| \leq C k^{p+1} .
$$

Moreover,

$$
\begin{aligned}
& \sum_{i=0}^{q} \beta_{i} B\left(u^{n+i}\right)-\sum_{i=0}^{q-1} \gamma_{i} B\left(u^{n+i}\right) \\
& \quad=\sum_{i=0}^{q} \beta_{i} \sum_{\ell=0}^{p-1} \frac{(i k)^{\ell}}{\ell !} \frac{\partial^{\ell}}{\partial t^{\ell}} B(u)\left(t_{n}\right)-\sum_{i=0}^{q-1} \gamma_{i} \sum_{\ell=0}^{p-1} \frac{(i k)^{\ell}}{\ell !} \frac{\partial^{\ell}}{\partial t^{\ell}} B(u)\left(t_{n}\right)+\varphi^{n} \\
& \quad=\sum_{\ell=0}^{p-1} \frac{k^{\ell}}{\ell !}\left(\sum_{i=0}^{q} \beta_{i} i^{\ell}-\sum_{i=0}^{q-1} \gamma_{i} i^{\ell}\right) \frac{\partial^{\ell}}{\partial t^{\ell}} B(u)\left(t_{n}\right)+\varphi^{n} \\
& =\varphi^{n}
\end{aligned}
$$

where the last equality holds in view of the consistency properties of $(\alpha, \beta)$ and $(\alpha, \gamma)$, and, obviously, $\left|\varphi^{n}\right| \leq c k^{p}$. This relation and (3.5i,ii) yield

$$
\max _{0 \leq n \leq N-q}\left|E_{1}^{n}\right| \leq C\left(k^{p}+h^{r}\right) .
$$

Finally, using (1.6), we obtain

$$
\max _{0 \leq n \leq N-q}\left\|E_{2}^{n}\right\|_{\star} \leq C h^{r},
$$

which completes the estimate of $E^{n}$.

Convergence of the multistep scheme. Let $\vartheta^{n}=W^{n}-U^{n}, n=0, \ldots, N$. Then (3.3) and (1.3) yield the error equation for $\vartheta^{n}$

$$
\sum_{i=0}^{q} \alpha_{i} \vartheta^{n+i}+k \sum_{i=0}^{q} \beta_{i} A_{h} \vartheta^{n+i}=k \sum_{i=0}^{q-1} \gamma_{i}\left\{B_{h}\left(W^{n+i}\right)-B_{h}\left(U^{n+i}\right)\right\}+k E^{n} .
$$

According to the splitting of $E^{n}$, we introduce $\vartheta_{1}^{j}$ and $\vartheta_{2}^{j}$, cf. section 2 , by

$$
\begin{aligned}
\sum_{i=0}^{q} \alpha_{i} \vartheta_{j}^{n+i}+k \sum_{i=0}^{q} \beta_{i} A_{h} \vartheta_{j}^{n+i}= & k \sum_{i=0}^{q-1} \gamma_{i} \int_{0}^{1} B_{h}^{\prime}\left(W^{n+i}-s \vartheta^{n+i}\right) d s \vartheta_{j}^{n+i} \\
& +k E_{j}^{n}, \quad j=1,2, n=0, \ldots, N-q,
\end{aligned}
$$

with initial values $\vartheta_{1}^{j}=\vartheta^{j}$ and $\vartheta_{2}^{j}=0$ for $j=0, \ldots, q-1$. Then, obviously, $\vartheta^{n}=\vartheta_{1}^{n}+\vartheta_{2}^{n}$. 
In the sequel we shall use the notation

$$
\begin{gathered}
\Theta_{j}^{n}=\left(\begin{array}{c}
\vartheta_{j}^{n+q-1} \\
\vdots \\
\vartheta_{j}^{n}
\end{array}\right), \mathcal{E}_{j}^{n}=\left(\begin{array}{c}
E_{j}^{n} \\
0 \\
\vdots \\
0
\end{array}\right), \quad \delta_{i}(x)=-\frac{\alpha_{i}+\beta_{i} x}{\alpha_{q}+\beta_{q} x}, \\
\Delta_{i}=\delta_{i}\left(k A_{h}\right), \quad \Gamma_{n}^{i}=\gamma_{i} \int_{0}^{1} B_{h}^{\prime}\left(W^{n+i}-s \vartheta^{n+i}\right) d s, \\
\Lambda=\Lambda\left(k A_{h}\right)=\left(\begin{array}{cccc}
\Delta_{q-1} & \Delta_{q-2} & \ldots & \Delta_{0} \\
I & 0 & & 0 \\
& \ddots & \ddots & \\
0 & & I & 0
\end{array}\right), \quad \Gamma_{n}=\left(\begin{array}{ccc}
\Gamma_{n}^{q-1} & \ldots & \Gamma_{n}^{0} \\
0 & \ldots & 0 \\
\vdots & & \vdots \\
0 & \ldots & 0
\end{array}\right),
\end{gathered}
$$

and

$$
\left(\alpha_{q}+k \beta_{q} A_{h}\right) \Theta_{j}^{n}=\left(\begin{array}{c}
\left(\alpha_{q}+k \beta_{q} A_{h}\right) \vartheta_{j}^{n+q-1} \\
\vdots \\
\left(\alpha_{q}+k \beta_{q} A_{h}\right) \vartheta_{j}^{n}
\end{array}\right) .
$$

Equation (3.9) can then be written in the form

$$
\left(\alpha_{q}+k \beta_{q} A_{h}\right) \Theta_{j}^{n+1}=\left(\alpha_{q}+k \beta_{q} A_{h}\right) \Lambda \Theta_{j}^{n}+k \Gamma_{n} \Theta_{j}^{n}+k \mathcal{E}_{j}^{n}, \quad j=1,2 .
$$

We quote the following result from Crouzeix, [3]:

Lemma 3.1. There exist a constant $\eta$, with $0 \leq \eta<1$, and a continuous map $\mathcal{H}: \overline{\mathbb{R}}^{+} \rightarrow \mathbb{C}^{q \times q}$ such that for all $x \geq 0$ the matrix $\mathcal{H}(x)$ is invertible and the Euclidean norm $\|\cdot\|_{2}$ of the matrix $\mathcal{L}(x)$,

$$
\mathcal{L}(x)=\frac{\alpha_{q}+\beta_{q} x}{\alpha_{q}+\eta \beta_{q} x} \mathcal{H}(x)^{-1} \Lambda(x) \mathcal{H}(x),
$$

is less or equal to one for all $x \geq 0$.

Let

$$
\mathcal{H}=\mathcal{H}\left(k A_{h}\right), \quad \mathcal{L}=\mathcal{L}\left(k A_{h}\right),
$$

and

$$
Y_{j}^{n}=\mathcal{H}^{-1} \Theta_{j}^{n}, \widetilde{\Gamma}_{n}=\mathcal{H}^{-1} \Gamma_{n}, \widetilde{\mathcal{E}}_{j}^{n}=\mathcal{H}^{-1} \mathcal{E}_{j}^{n}
$$

then, we can rewrite $(3.10)$ as

$$
\left(\alpha_{q}+k \beta_{q} A_{h}\right) Y_{j}^{n+1}=\left(\alpha_{q}+k \eta \beta_{q} A_{h}\right) \mathcal{L} Y_{j}^{n}+k \widetilde{\Gamma}_{n} \mathcal{H} Y_{j}^{n}+k \widetilde{\mathcal{E}}_{j}^{n}, \quad j=1,2 .
$$

In view of the fact that $\|\mathcal{H}(x)\|_{2},\left\|\mathcal{H}(x)^{-1}\right\|_{2}$ are uniformly bounded, see relations (3.27) and (3.28) in [3], it suffices to estimate $Y^{n}$. We adjust in this section the definition of $\|\cdot\|$ to the scheme under consideration by setting

$$
\|v\|:=\left(\alpha_{q}|v|^{2}+\beta_{q} k\|v\|^{2}\right)^{1 / 2}, \quad v \in V .
$$


Further, for $V=\left(v_{1}, \ldots, v_{q}\right)^{T}$ and $W=\left(w_{1}, \ldots, w_{q}\right)^{T}$ in $H^{q}$ or in $V^{q}$ we shall use the notation

$$
\begin{gathered}
(V, W):=\sum_{i=1}^{q}\left(v_{i}, w_{i}\right), \quad|V|:=\left(\sum_{i=1}^{q}\left|v_{i}\right|^{2}\right)^{1 / 2} \\
\|V\|:=\left(\sum_{i=1}^{q}\left\|v_{i}\right\|^{2}\right)^{1 / 2}, \quad\|V\|:=\left(\sum_{i=1}^{q}\left\|v_{i}\right\|^{2}\right)^{1 / 2}, \quad\|V\|_{\star}:=\left(\sum_{i=1}^{q}\left\|v_{i}\right\|_{\star}^{2}\right)^{1 / 2}
\end{gathered}
$$

and, for a linear operator $M: H^{q} \rightarrow H^{q}$, we set $|M|:=\sup _{V \in H^{q}, V \neq 0} \frac{|M V|}{|V|}$.

The main result in this paper is given in the following theorem:

Theorem 3.1. Assume that the constant $\lambda$ in (1.4) is appropriately small (depending on the particular scheme) and that $k$ and $h^{2 r} k^{-1}$ are sufficiently small. Then, we have the local stability estimate

$$
\begin{aligned}
\left|\vartheta^{n}\right|+k^{1 / 2}\left\|\vartheta^{n}\right\| \leq & C e^{c m T}\left\{\sum_{j=0}^{q-1}\left(\left|\vartheta^{j}\right|+k^{1 / 2}\left\|\vartheta^{j}\right\|\right)+k \sum_{j=0}^{n-q}\left|E_{1}^{j}\right|\right. \\
& \left.+\left(k \sum_{j=0}^{n-q}\left\|E_{2}^{j}\right\|_{\star}^{2}\right)^{1 / 2}\right\}, \quad n=q-1, \ldots, N,
\end{aligned}
$$

and the error estimate

$$
\max _{0 \leq n \leq N}\left|u\left(t_{n}\right)-U^{n}\right| \leq C\left(k^{p}+h^{r}\right) .
$$

Proof. Let $\rho^{n}=u^{n}-W^{n}, n=0, \ldots, N$. Then, according to (1.5),

$$
\max _{0 \leq n \leq N}\left|\rho^{n}\right| \leq C h^{r}
$$

Now, if we assume that (3.12) holds, using (3.2), (3.6) and (3.7), we obtain

$$
\max _{0 \leq n \leq N}\left|\vartheta^{n}\right| \leq C\left(k^{p}+h^{r}\right),
$$

and (3.13) follows immediately from (3.14) and (3.15). Thus, it remains to prove (3.12). According to (3.2), (3.6) and (3.7), there exists a constant $C_{\star}$ such that the right-hand side of (3.12) can be estimated by $C_{\star}\left(k^{p}+h^{r}\right)$,

$$
\begin{gathered}
C e^{c m T}\left\{\sum_{j=0}^{q-1}\left(\left|\vartheta^{j}\right|+k^{1 / 2}\left\|\vartheta^{j}\right\|\right)+k \sum_{j=0}^{n-q}\left|E_{1}^{j}\right|\right. \\
\left.+\left(k \sum_{j=0}^{N-q}\left\|E_{2}^{j}\right\|_{\star}^{2}\right)^{1 / 2}\right\} \\
\leq C_{\star}\left(k^{p}+h^{r}\right) .
\end{gathered}
$$

We will estimate $\vartheta^{n}$ by estimating $Y_{j}^{n}$. In fact, we shall show that for some $\varepsilon=$ $\varepsilon(\lambda,(\alpha, \beta, \gamma)), 0<\varepsilon<\left(1-\eta^{2}\right) \beta_{q}$ with $\eta$ as in Lemma 3.1,

$$
\left\|Y_{1}^{n}\right\| \leq e^{c m t_{n}}\left\{\left\|Y_{1}^{0}\right\|+\frac{k}{\sqrt{\alpha_{q}}} \sum_{j=0}^{n-1}\left|\widetilde{\mathcal{E}}_{1}^{j}\right|\right\}
$$

and

$$
\left\|Y_{2}^{n}\right\| \leq e^{c m t_{n}} \frac{1}{\sqrt{\varepsilon}}\left(k \sum_{j=0}^{n-1}\left\|\widetilde{\mathcal{E}}_{2}^{j}\right\|_{\star}^{2}\right)^{1 / 2} .
$$

Then, (3.12) follows, and the proof will be complete. We shall use induction: The estimates (3.17) and (3.18) are valid for $n=0$. Assume that they hold for $0, \ldots, n$, 
$0 \leq n \leq N-q$. Then, according to (3.16) and (3.12), which is then valid for $0, \ldots, n+q-1$, we have, for $k$ and $k^{-1} h^{2 r}$ small enough,

$$
\max _{0 \leq j \leq n+q-1}\left\|\vartheta^{j}\right\| \leq C_{\star}\left(k^{p-1 / 2}+k^{-1 / 2} h^{r}\right) \leq 1,
$$

i.e.,

$$
\sup _{0<s<1} \max _{0 \leq j \leq n+q-1} \mu\left(W^{j}-s \vartheta^{j}\right) \leq m .
$$

Taking in (3.11) the inner product with $Y_{j}^{n+1}$, we have

$$
\begin{aligned}
\left\|Y_{j}^{n+1}\right\|^{2}=\left(\left(\alpha_{q}\right.\right. & \left.\left.+k \eta \beta_{q} A_{h}\right) \mathcal{L} Y_{j}^{n}, Y_{j}^{n+1}\right)+k\left(\widetilde{\Gamma}_{n} \mathcal{H} Y_{j}^{n}, Y_{j}^{n+1}\right) \\
& +k\left(\widetilde{\mathcal{E}}_{j}^{n}, Y_{j}^{n+1}\right), \quad j=1,2 .
\end{aligned}
$$

First, we shall estimate the second term on the right-hand side of (3.20). Let $X, Y \in V^{q}$ and $X^{\prime}=\mathcal{H}^{-1} X, Y^{\prime}=\mathcal{H}^{T} Y$, and recall that $\widetilde{\Gamma}_{n}=\mathcal{H}^{-1} \Gamma_{n}$. Then, if $x_{i}, y_{i}$ are the components of $X$ and $Y$, respectively, we have

$$
\left(\widetilde{\Gamma}_{n} X, Y^{\prime}\right)=\left(\Gamma_{n} X, Y\right)=\sum_{i=0}^{q-1} \gamma_{i} \int_{0}^{1}\left(B_{h}^{\prime}\left(W^{n+i}-s \vartheta^{n+i}\right) x_{i}, y_{1}\right) d s .
$$

Using here the induction hypothesis, which ensures (3.19), the assumption (1.4) and the fact that $\|\mathcal{H}(x)\|_{2}$ and $\left\|\mathcal{H}(x)^{-1}\right\|_{2}$ are uniformly bounded, we see that there exists a constant $M_{1}$ such that

$$
\left|\left(\widetilde{\Gamma}_{n} X, Y^{\prime}\right)\right| \leq M_{1}\left(\lambda \left\|X ^ { \prime } \left|\left\||Y \|+m| X^{\prime}|| Y \mid\right) .\right.\right.\right.
$$

Therefore,

$$
\left|\left(\widetilde{\Gamma}_{n} \mathcal{H} Y_{j}^{n}, Y_{j}^{n+1}\right)\right| \leq M_{1}\left(\lambda\left\|Y_{j}^{n}\right\|\left\|Y_{j}^{n+1}\right\|+m\left|Y_{j}^{n}\right|\left|Y_{j}^{n+1}\right|\right), \quad j=1,2 .
$$

Next, we shall estimate the first term on the right-hand side of (3.20). Lemma 3.1 implies that

$$
|\mathcal{L}| \leq 1
$$

We also have

$$
\left(\left(\alpha_{q}+k \eta \beta_{q} A_{h}\right) \mathcal{L} Y_{j}^{n}, Y_{j}^{n+1}\right)=\alpha_{q}\left(\mathcal{L} Y_{j}^{n}, Y_{j}^{n+1}\right)+k \eta \beta_{q}\left(\mathcal{L}\left(A_{h}^{1 / 2} Y_{j}^{n}\right), A_{h}^{1 / 2} Y_{j}^{n+1}\right),
$$

i.e., in view of (3.22),

$$
\left|\left(\left(\alpha_{q}+k \eta \beta_{q} A_{h}\right) \mathcal{L} Y_{j}^{n}, Y_{j}^{n+1}\right)\right| \leq \alpha_{q}\left|Y_{j}^{n}\right|\left|Y_{j}^{n+1}\right|+\eta \beta_{q} k\left\|Y_{j}^{n}\right\|\left\|Y_{j}^{n+1}\right\|,
$$

From (3.20), (3.21) and (3.23), we obtain

$$
\begin{aligned}
\left\|Y_{j}^{n+1}\right\|^{2} \leq \alpha_{q}\left|Y_{j}^{n}\right|\left|Y_{j}^{n+1}\right|+\left(\lambda M_{1}+\eta \beta_{q}\right) k\left\|Y_{j}^{n}\right\|\left\|Y_{j}^{n+1}\right\| \\
+M_{1} m k\left|Y_{j}^{n}\right|\left|Y_{j}^{n+1}\right|+k\left(\widetilde{\mathcal{E}}_{j}^{n}, Y_{j}^{n+1}\right), \quad j=1,2 .
\end{aligned}
$$

Therefore, with $\lambda=\frac{(1-\eta) \beta_{q}}{M_{1}}$ and $c=\frac{M_{1}}{\alpha_{q}}$,

$$
\begin{aligned}
\left\|Y_{1}^{n+1}\right\|^{2} & \leq(1+c m k) \alpha_{q}\left|Y_{1}^{n}\right|\left|Y_{1}^{n+1}\right|+\beta_{q} k\left\|Y_{1}^{n}\right\|\left\|Y_{1}^{n+1}\right\|+k\left|\widetilde{\mathcal{E}}_{1}^{n}\right|\left|Y_{1}^{n+1}\right| \\
& \leq(1+c m k)\left(\alpha_{q}\left|Y_{1}^{n}\right|\left|Y_{1}^{n+1}\right|+\beta_{q} k\left\|Y_{1}^{n}\right\|\left\|Y_{1}^{n+1}\right\|\right)+k\left|\widetilde{\mathcal{E}}_{1}^{n}\right|\left|Y_{1}^{n+1}\right|,
\end{aligned}
$$

i.e.,

$$
\left\|Y_{1}^{n+1}\right\| \leq(1+c m k)\left\|Y_{1}^{n}\right\|+\frac{k}{\sqrt{\alpha_{q}}}\left|\widetilde{\mathcal{E}}_{1}^{n}\right| .
$$


From (3.25) and the induction hypothesis, it easily follows that (3.17) holds also for $n+1$. Similarly, with $\lambda=\frac{1}{M_{1}}\left\{\left[\beta_{q}\left(\beta_{q}-\varepsilon\right)\right]^{1 / 2}-\eta \beta_{q}\right\}$ for some $\varepsilon, 0<\varepsilon<\left(1-\eta^{2}\right) \beta_{q}$, and $c=\frac{M_{1}}{\alpha_{q}}$,

$$
\begin{gathered}
\left\|Y_{2}^{n+1}\right\|^{2} \leq(1+c m k) \alpha_{q}\left|Y_{2}^{n}\right|\left|Y_{2}^{n+1}\right|+\left[\beta_{q}\left(\beta_{q}-\varepsilon\right)\right]^{1 / 2} k\left\|Y_{2}^{n}\right\|\left\|Y_{2}^{n+1}\right\| \\
+\frac{1}{2 \varepsilon} k\left\|\widetilde{\mathcal{E}}_{2}^{n}\right\|_{\star}^{2}+\frac{\varepsilon}{2} k\left\|Y_{2}^{n+1}\right\|^{2} .
\end{gathered}
$$

Thus,

$$
\begin{gathered}
\left\|Y_{2}^{n+1}\right\|^{2} \leq \frac{1}{2}(1+c m k)^{2} \alpha_{q}\left|Y_{2}^{n}\right|^{2}+\frac{1}{2} \alpha_{q}\left|Y_{2}^{n+1}\right|^{2}+\frac{1}{2} \beta_{q} k\left\|Y_{2}^{n}\right\|^{2} \\
+\frac{1}{2} \beta_{q} k\left\|Y_{2}^{n+1}\right\|^{2}+\frac{1}{2 \varepsilon} k\left\|\widetilde{\mathcal{E}}_{2}^{n}\right\|_{\star}^{2}
\end{gathered}
$$

i.e.,

$$
\left\|Y_{2}^{n+1}\right\|^{2} \leq(1+c m k)^{2}\left\|Y_{2}^{n}\right\|^{2}+\frac{k}{\varepsilon}\left\|\widetilde{\mathcal{E}}_{2}^{n}\right\|_{\star}^{2} .
$$

From (3.26) and the induction hypothesis, it easily follows that (3.18) holds for $n+1$ as well, and the proof is complete.

Remark 3.2. Initial approximations. Assume that the data of the problem are smooth enough such that one can compute the time derivatives $u^{(j)}(0), j=1, \ldots$, $p-1$, of the exact solution at $t=0$. Then, it is easily seen that $U^{0}=W^{0}$ and $U^{m}=R_{h} T_{m}^{p} u(0), m=1, \ldots, q-1$, with

$$
T_{m}^{p} u(0)=u^{0}+m k u^{(1)}(0)+\cdots+\frac{(m k)^{p-1}}{(p-1) !} u^{(p-1)}(0), \quad m=1, \ldots, q-1,
$$

satisfy $(3.2)$.

\section{Applications}

In this section we shall apply our abstract results to two examples in one space dimension, namely the periodic initial value problems for the Kuramoto-Sivashinsky equation and the Cahn-Hilliard equation, and to a class of reaction diffusion equations in $\mathbb{R}^{\nu}, \nu=2,3$.

4.1 The Kuramoto-Sivashinsky equation. We consider the periodic initial value problem for the Kuramoto-Sivashinsky (KS) equation: For $T, \nu>0$ we seek a real-valued function $u$ defined on $\mathbb{R} \times[0, T], 1$-periodic in the space variable and satisfying

$$
u_{t}+u u_{x}+u_{x x}+\nu u_{x x x x}=0 \quad \text { in } \quad \mathbb{R} \times[0, T]
$$

and

$$
u(\cdot, 0)=u^{0} \quad \text { in } \quad \mathbb{R},
$$

where $u^{0}$ is a given, smooth 1-periodic function.

The periodic initial value problem (4.1)-(4.2) is well-posed, cf. [13], [17]. For numerical methods to this problem see, e.g., [1] and the references therein. The KS equation is related to turbulence phenomena in chemistry and combustion, and arises also in plasma physics and in two-phase flows in cylindrical geometries, see [18] and [14]. 
For $s \in \mathbb{N}_{0}$, let $H_{\text {per }}^{s}$ denote the periodic Sobolev space of order $s$, consisting of the 1-periodic elements of $H_{\text {loc }}^{s}(\mathbb{R})$, and let $\|\cdot\|_{H^{s}}$ be the norm over a period in $H_{\mathrm{per}}^{s}$. The inner product in $H:=L_{\mathrm{per}}^{2}=H_{\mathrm{per}}^{0}$ is denoted by $(\cdot, \cdot)$, and the induced norm by $|\cdot|$. Let $A: H_{\text {per }}^{4} \rightarrow H$ be defined by $A v=\nu\left(v_{x x x x}+v\right)$. Then $V:=D\left(A^{1 / 2}\right)=H_{\text {per }}^{2}$, and the norm in $V$ is given by $\|v\|=\nu^{1 / 2}\left(\left|v_{x x}\right|^{2}+|v|^{2}\right)^{1 / 2}$. Let $B: V \rightarrow H$ be given by $B(v)=-v_{x x}-v v_{x}+\nu v$. Then,

$$
B^{\prime}(v) w=-w_{x x}-(v w)_{x}+\nu w,
$$

and thus by periodicity

$$
\left(B^{\prime}(v) w, \omega\right)=\left(w_{x}, \omega_{x}\right)+\left(v, w \omega_{x}\right)+\nu(w, \omega) .
$$

Therefore, in view of the inequality $\|w\|_{L^{\infty}} \leq|w|+\left|w^{\prime}\right|, w \in H_{\text {per }}^{1}$,

$$
\begin{aligned}
\left|\left(B^{\prime}(v) w, \omega\right)\right| & \leq\left|w_{x}\right|\left|\omega_{x}\right|+|v||| w \|_{L^{\infty}}\left|\omega_{x}\right|+\nu|w||\omega| \\
& \leq(1+|v|)\left|w_{x}\right|\left|\omega_{x}\right|+\nu|w||\omega|+|v||w|\left|\omega_{x}\right|,
\end{aligned}
$$

and thus, using the inequality $\left|u_{x}\right|^{2} \leq|u|\left|u_{x x}\right|, u \in V$, we easily see that the condition (1.4) is satisfied for any $\lambda>0$,

$$
\left|\left(B^{\prime}(v) w, \omega\right)\right| \leq \lambda\|w\|\|\omega\|+\mu(v)|w||\omega|, \quad \forall v, w, \omega \in V,
$$

with $\mu(v):=\frac{1}{2 \lambda \nu}\left[1+2 \lambda \nu^{2}+2|v|(1+|v|)\right]$. We note that, since $\lambda$ can be taken arbitrarily small, our results hold for this problem for all implicit-explicit schemes satisfying our stability and consistency assumptions; further, since $\mu$ is bounded for $v$ bounded in $H$, the meshcondition of Theorems 2.1 and 3.1 is not needed here, cf. Remark 2.1.

For the space discretization, we let $0=x_{0}<x_{1}<\cdots<x_{J}=1$ be a partition of $[0,1]$, and $h:=\max _{j}\left(x_{j+1}-x_{j}\right)$. Setting $x_{j J+s}:=j+x_{s}, j \in \mathbb{Z}, s=0, \ldots, J-1$, this partition is periodically extended to a partition of $\mathbb{R}$. For integer $r \geq 4$, let $V_{h}$ denote a space of at least once continuously differentiable, 1-periodic splines of degree $r-1$, in which approximations to the solution $u(\cdot, t)$ of $(4.1)-(4.2)$ will be sought for $0 \leq t \leq T$. The following approximation property of the family $\left\{V_{h}\right\}_{0<h<1}$ is well known, cf., e.g., [16],

$$
\inf _{\chi \in V_{h}} \sum_{j=0}^{2} h^{j}\|v-\chi\|_{H^{j}} \leq c h^{s}\|v\|_{H^{s}}, \quad v \in H_{\mathrm{per}}^{s}, \quad 2 \leq s \leq r .
$$

We define the elliptic projection operator $R_{h}: V \rightarrow V_{h}$ by

$$
\left(\left(v-R_{h} v\right)^{\prime \prime}, \chi^{\prime \prime}\right)+\left(v-R_{h} v, \chi\right)=0 \quad \forall \chi \in V_{h} .
$$

It is easily seen that

$$
\sum_{j=0}^{2} h^{j}\left\|v-R_{h} v\right\|_{j} \leq c h^{s}\|v\|_{H^{s}}, \quad v \in H_{\mathrm{per}}^{s}, \quad 2 \leq s \leq r ;
$$

thus, in particular, the estimate (1.5) holds in this case with $d=4$. Next, we will verify (1.6). For $v, \omega \in V$, and $w:=R_{h} v$, we have

$$
(B(v)-B(w), \omega)=-\left(v-w, \omega^{\prime \prime}\right)+\frac{1}{2}\left((v+w)(v-w), \omega^{\prime}\right)+\nu(v-w, \omega),
$$

and, by (4.6),

$$
|(B(v)-B(w), \omega)| \leq C\left(1+\|v\|_{H^{2}}\right)\|v\|_{H^{r}} h^{r}\|\omega\|,
$$


i.e.,

$$
\left\|B(v)-B\left(R_{h} v\right)\right\|_{\star} \leq C\left(1+\|v\|_{H^{2}}\right)\|v\|_{H^{r}} h^{r},
$$

and thus (1.6) holds. Let $W(t):=R_{h} u(t)$, and assume that we are given approximations $U^{0}, \ldots, U^{q-1} \in V_{h}$ to $u^{0}, \ldots, u^{q-1}$ such that

$$
\sum_{j=0}^{q-1}\left(\left|W^{j}-U^{j}\right|+k^{1 / 2}\left\|W^{j}-U^{j}\right\|\right) \leq c\left(k^{p}+h^{r}\right) .
$$

Then, we define $U^{n} \in V_{h}, n=q, \ldots, N$, recursively by the $(\alpha, \beta, \gamma)$ scheme

$$
\begin{gathered}
\sum_{i=0}^{q} \alpha_{i}\left(U^{n+i}, \chi\right)+k \nu \sum_{i=0}^{q} \beta_{i}\left\{\left(U_{x x}^{n+i}, \chi^{\prime \prime}\right)+\left(U^{n+i}, \chi\right)\right\} \\
=k \sum_{i=0}^{q-1} \gamma_{i}\left\{\left(U_{x}^{n+i}, \chi^{\prime}\right)-\left(U^{n+i} U_{x}^{n+i}, \chi\right)+\nu\left(U^{n+i}, \chi\right)\right\} \\
\forall \chi \in V_{h}, \quad n=0, \ldots, N-q,
\end{gathered}
$$

where $(\alpha, \beta)$ and $(\alpha, \gamma)$ are multistep schemes of order $p$, and $(\alpha, \beta)$ is strongly $A(0)$-stable. Then, Theorem 3.1 yields, for sufficiently small $k$ and $h$, the error estimate

$$
\max _{n}\left|u^{n}-U^{n}\right| \leq c\left(k^{p}+h^{r}\right) .
$$

As already mentioned, (4.10) holds for all $(\alpha, \beta, \gamma)$ schemes satisfying our stability and consistency properties.

Remark 4.1. It is not difficult to verify that the estimate (4.10) remains valid if the approximations $U^{n} \in V_{h}, n=q, \ldots, N$, are defined by

$$
\begin{gathered}
\sum_{i=0}^{q} \alpha_{i}\left(U^{n+i}, \chi\right)+k \nu \sum_{i=0}^{q} \beta_{i}\left(U_{x x}^{n+i}, \chi^{\prime \prime}\right) \\
=k \sum_{i=0}^{q-1} \gamma_{i}\left\{\left(U_{x}^{n+i}, \chi^{\prime}\right)-\left(U^{n+i} U_{x}^{n+i}, \chi\right)\right\} \\
\forall \chi \in V_{h}, \quad n=0, \ldots, N-q .
\end{gathered}
$$

4.2 The Cahn-Hilliard equation. We consider the periodic initial value problem for the Cahn-Hilliard $(\mathrm{CH})$ equation: For $T>0$ we seek a real-valued function $u$ defined on $\mathbb{R} \times[0, T], 1$-periodic in the space variable and satisfying

$$
u_{t}+u_{x x x x}-\left(u^{3}-u\right)_{x x}=0 \quad \text { in } \quad \mathbb{R} \times[0, T]
$$

and

$$
u(\cdot, 0)=u^{0} \quad \text { in } \quad \mathbb{R},
$$

where $u^{0}$ is a given, smooth 1-periodic function.

The problem (4.11)-(4.12) is well-posed, see Temam [18] and the references therein. For numerical methods for this problem we refer to, e.g., [6], [12]. The $\mathrm{CH}$ equation describes the dynamics of pattern formation through phase transition, cf., e.g., [18].

We shall use the same Hilbert spaces, and approximating spaces as in subsection 4.1. We let $A: H_{\text {per }}^{4} \rightarrow H$ be defined by $A v=v_{x x x x}+v$. Thus, the norm in $V$, 
$V:=D\left(A^{1 / 2}\right)=H_{\text {per }}^{2}$, is given by $\|v\|:=\left(\left|v_{x x}\right|^{2}+|v|^{2}\right)^{1 / 2}$. Let $B: V \rightarrow H$ be given by $B(v)=\left(v^{3}-v\right)_{x x}+v$. Then,

$$
B^{\prime}(v) w=3\left(v^{2} w\right)_{x x}-w_{x x}+w .
$$

In this case we can show that

$$
\left|\left(B^{\prime}(v) w, \omega\right)\right| \leq \lambda\|w\|\|\omega\|+\mu(v)|w||\omega|, \quad \forall v, w, \omega \in V,
$$

with $\mu(v):=1+\frac{1}{2 \sqrt{2} \lambda}\left\{\left(1+3\|v\|_{L^{\infty}}^{2}+6\|v\|_{L^{\infty}}\left|v_{x}\right|\right)^{2}+36\|v\|_{L^{\infty}}^{2}\left|v_{x}\right|^{2}\right\}$. We note that $\mu$ is bounded for $v$ bounded in $H_{\text {per }}^{1}$. Further, since

$$
\|v\|_{H^{1}} \leq|v|+|v|^{1 / 2}\|v\|^{1 / 2}, \quad \forall v \in V
$$

we conclude that a condition of the form (2.23) holds in this case with $a=\frac{1}{2}$.

The projection $R_{h}$ is also in this case defined by (4.5). In particular, in view of (4.6) the condition (1.5) is satisfied. Moreover, it is easily seen that

$$
\left\|B(v)-B\left(R_{h} v\right)\right\|_{\star} \leq C\left(1+\|v\|_{H^{2}}^{2}\right)\|v\|_{H^{r}} h^{r},
$$

and thus (1.6) holds in this case. Let $W(t):=R_{h} u(t)$ and assume that we are given approximations $U^{0}, U^{1}, \ldots, U^{q-1} \in V_{h}$ satisfying (4.8). Then we define $U^{n} \in V_{h}$, $n=q, \ldots, N$, recursively by the $(\alpha, \beta, \gamma)$ scheme

$$
\begin{gathered}
\sum_{i=0}^{q} \alpha_{i}\left(U^{n+i}, \chi\right)+k \sum_{i=0}^{q} \beta_{i}\left\{\left(U_{x x}^{n+i}, \chi^{\prime \prime}\right)+\left(U^{n+i}, \chi\right)\right\} \\
=k \sum_{i=0}^{q-1} \gamma_{i}\left\{\left(\left(U^{n+i}\right)^{3}-U^{n+i}, \chi^{\prime \prime}\right)+\left(U^{n+i}, \chi\right)\right\} \\
\forall \chi \in V_{h}, \quad n=0, \ldots, N-q,
\end{gathered}
$$

where $(\alpha, \beta)$ and $(\alpha, \gamma)$ are multistep schemes of order $p$, and $(\alpha, \beta)$ is strongly $A(0)$-stable. Then, taking into account (4.14) and Remark 2.1, an application of Theorem 3.1 yields, for $k$ and $h^{4 r} k^{-1}$ sufficiently small, the error estimate

$$
\max _{n}\left|u^{n}-U^{n}\right| \leq c\left(k^{p}+h^{r}\right) .
$$

4.3 A reaction diffusion equation. In this subsection we shall apply our results to a class of reaction diffusion equations: Let $\Omega \subset \mathbb{R}^{\nu}, \nu=2,3$, be a bounded domain with smooth boundary $\partial \Omega$. For $T>0$ we seek a real-valued function $u$, defined on $\bar{\Omega} \times[0, T]$, satisfying

$$
\begin{array}{ll}
u_{t}-\Delta u=f(u) & \text { in } \Omega \times[0, T], \\
u=0 & \text { on } \partial \Omega \times[0, T], \\
u(\cdot, 0)=u^{0} & \text { in } \Omega,
\end{array}
$$

where $u^{0}$ is a given smooth function and $f: \mathbb{R} \rightarrow \mathbb{R}$ is a smooth function. We are interested in approximating smooth solutions of this problem, and assume therefore that the data are smooth and compatible such that (4.18) gives rise to sufficiently regular solutions.

We shall distinguish two cases. Assuming that $f$ satisfies a polynomial growth condition of order at most $\rho$, see (4.19) below, and provided that $\rho \leq 4$ for $\nu=3$, we show that the abstract theory of sections 2 and 3 is directly applicable and yields, without any additional assumptions on the discretization spaces, optimal order error estimates for all $(\alpha, \beta, \gamma)$ schemes considered in this paper. For general 
$f$ our results apply as well, provided that meshconditions stronger than those of Theorem 3.1 are fulfilled.

Reaction diffusion equations model various physical phenomena related, for instance, to phase transitions, chemical reactions, pattern formation, cf., e.g., [2], [7], [10] and their references. The Allen-Cahn model $(\rho=3)$, which in the limit describes evolution by mean curvature, [2], [7], and generalized Ginzburg-Landau equations, [10], are reaction diffusion equations of particular interest with polynomial nonlinearity.

Let $H^{s}=H^{s}(\Omega)$ be the usual Sobolev space of order $s$, and $\|\cdot\|_{H^{s}}$ be the norm of $H^{s}$. The inner product in $H:=L^{2}(\Omega)$ is denoted by $(\cdot, \cdot)$, and the induced norm by $|\cdot|$; the norm of $L^{s}(Q), 1 \leq s \leq \infty$, is denoted by $\|\cdot\|_{L^{s}(Q)}$ and simply by $\|\cdot\|_{L^{s}}$ for $Q=\Omega$. Obviously, $V=H_{0}^{1}(\Omega)=H_{0}^{1}$ and the norm in $V$ is equivalent to the $H^{1}$ norm.

We now consider the case that $f$ satisfies the following growth condition: there exists $L \in \mathbb{R}$ such that

$$
\left|f^{\prime}(\xi)\right| \leq L\left(1+|\xi|^{\rho-1}\right) \quad \forall \xi \in \mathbb{R} .
$$

In the sequel, we shall use the Sobolev inequality

$$
\|v\|_{L^{s}} \leq C\|v\|_{H^{1}}, \quad 1 \leq s<\infty \text { for } \nu=2, \quad \text { and } 1 \leq s \leq 6 \text { for } \nu=3,
$$

as well as its consequence

$$
\|v\|_{L^{4}} \leq C\|v\|_{L^{2}}^{1-\nu / 4}\|v\|_{H^{1}}^{\nu / 4}, \quad \nu=2,3 .
$$

Let $B: V \rightarrow V^{\prime}, B(v)=f(v)$. First, we note that $B$ is well defined. Indeed, for $v, w \in V$,

$$
(f(v), w)=\left(\int_{0}^{1} f^{\prime}(s v) v d s, w\right)+(f(0), w),
$$

and, therefore, by (4.19) and Hölder's inequality,

$$
|(f(v), w)| \leq C\left[\left(\int_{\Omega}|v|^{\rho 6 / 5} d x\right)^{5 / 6}\|w\|_{L^{6}}+\left(1+\|v\|_{L^{2}}\right)\|w\|_{L^{2}}\right],
$$

and thus, in view of (4.20), we see that $f(v) \in V^{\prime}$, provided $\rho \leq 5$ for $\nu=3$. Further, by (4.19), for $v, w, \omega \in V$,

$$
\begin{aligned}
\left|\left(f^{\prime}(v) w, \omega\right)\right| & \leq C \int_{\Omega}|v|^{\rho-1}|w||\omega| d x+C\|w\|_{L^{2}}\|\omega\|_{L^{2}} \\
& \leq C\left(\int_{\Omega}|v|^{2(\rho-1)} d x\right)^{1 / 2}\|w\|_{L^{4}}\|\omega\|_{L^{4}}+C\|w\|_{L^{2}}\|\omega\|_{L^{2}}
\end{aligned}
$$

i.e., in view of (4.21),

$$
\left|\left(f^{\prime}(v) w, \omega\right)\right| \leq C\|v\|_{L^{2(\rho-1)}}^{\rho-1}|w|^{\frac{4-\nu}{4}}|\omega|^{\frac{4-\nu}{4}}\|w\|^{\frac{\nu}{4}}\|\omega\|^{\frac{\nu}{4}}+C|w||\omega| .
$$

Thus, $B$ is differentiable. Furthermore, (4.23) and Young's inequality ( $a b \leq \frac{\varepsilon}{q} a^{q}+$ $\left.\frac{\varepsilon^{-q^{\prime} / q}}{q^{\prime}} b^{q^{\prime}}, \frac{1}{q}+\frac{1}{q^{\prime}}=1\right)$ yield

$$
\begin{aligned}
\left|\left(B^{\prime}(v) w, \omega\right)\right| \leq C & \frac{\nu}{4} \varepsilon\|w\|\|\omega\| \\
& +C\left(1+\frac{4-\nu}{4} \varepsilon^{-\frac{\nu}{4-\nu}}\left(\|v\|_{L^{2(\rho-1)}}\right)^{\frac{4(\rho-1)}{4-\nu}}\right)|w||\omega| .
\end{aligned}
$$


Therefore, by Sobolev's inequality, we see that, for $\rho \leq 4$ when $\nu=3$ (and for any $\rho$ when $\nu=2$ ), the assumption (1.4) is satisfied with $\lambda=C \frac{\nu}{4} \varepsilon$,

$$
\mu(v)=C+C \frac{4-\nu}{4} \varepsilon^{-\frac{\nu}{4-\nu}}\left(\|v\|_{L^{2(\rho-1)}}\right)^{\frac{4(\rho-1)}{4-\nu}},
$$

and $\mu(v)$ is bounded for $v$ bounded in $V$. Again, $\lambda$ can be taken arbitrarily small and thus our theory applies to all $(\alpha, \beta, \gamma)$ schemes satisfying our stability and consistency assumptions.

We further analyze the case that $f$ satisfies the growth condition (4.19), with $\rho \leq 4$ for $\nu=3$, by introducing the discretization spaces $V_{h}$; the case of general $f$ shall be discussed at the end of this section. For simplicity, let $V_{h}$ be the subspace of $V$ defined on a finite element partition $\mathcal{T}_{h}$ of $\Omega$, and consisting of piecewise polynomial functions of degree at most $r-1, r \geq 2$. Let $h_{K}$ denote the diameter of an element $K \in \mathcal{T}_{h}$, and $h=\max _{K \in \mathcal{T}_{h}} h_{K}$. We define the elliptic projection operator $R_{h}, R_{h}: V \rightarrow V_{h}$, by

$$
\left(\nabla R_{h} v, \nabla \chi\right)=(\nabla v, \nabla \chi) \quad \forall \chi \in V_{h} .
$$

We assume that (we do not attempt here to deal with problems that may arise in the case of a curved boundary $\partial \Omega$ concerning the requirement $V_{h} \subset V$, cf. Remark $2.2)$

$$
\left|v-R_{h} v\right|+h\left\|v-R_{h} v\right\| \leq C h^{r}\|v\|_{H^{r}}, \quad v \in H^{r} \cap H_{0}^{1} ;
$$

then, in particular, the estimate (1.5) will hold in this case with $d=2$. Next, we will verify (1.6). We shall further assume that

$$
\sup \left\{\left\|R_{h} v\right\|_{L^{\infty}}: 0<h<1\right\} \leq C\|v\|_{H^{r}}, \quad v \in H^{r} \cap H_{0}^{1} .
$$

For $v \in H^{r} \cap H_{0}^{1}$, we have

$$
B(v)-B\left(R_{h} v\right)=\int_{0}^{1} f^{\prime}\left(v-\tau\left(v-R_{h} v\right)\right) d \tau\left(v-R_{h} v\right) ;
$$

using here the Sobolev inequality $\|v\|_{L^{\infty}} \leq C\|v\|_{H^{r}}(r \geq 2)$ and (4.26), we conclude, in view of (4.25),

$$
\left|B(v)-B\left(R_{h} v\right)\right| \leq C h^{r} \tilde{M}(v)\|v\|_{H^{r}},
$$

with $\tilde{M}(v)$ bounded for $v \in H^{r} \cap H_{0}^{1}$ bounded in $H^{r}$. Thus, (1.6) is satisfied.

Now, let $W(t):=R_{h} u(t)$, and assume that we are given approximations $U^{0}, \ldots$, $U^{q-1} \in V_{h}$ to $u^{0}, \ldots, u^{q-1}$ such that

$$
\sum_{j=0}^{q-1}\left(\left|W^{j}-U^{j}\right|+k^{1 / 2}\left\|W^{j}-U^{j}\right\|\right) \leq c\left(k^{p}+h^{r}\right) .
$$

Then, we define $U^{n} \in V_{h}, n=q, \ldots, N$, recursively by the $(\alpha, \beta, \gamma)$ scheme

$$
\begin{aligned}
& \sum_{i=0}^{q} \alpha_{i}\left(U^{n+i}, \chi\right)+k \sum_{i=0}^{q} \beta_{i}\left(\nabla U^{n+i}, \nabla \chi\right) \\
&=k \sum_{i=0}^{q-1} \gamma_{i}\left(f\left(U^{n+i}\right), \chi\right) \quad \forall \chi \in V_{h}, \quad n=0, \ldots, N-q,
\end{aligned}
$$


where $(\alpha, \beta)$ and $(\alpha, \gamma)$ are multistep schemes of order $p$, and $(\alpha, \beta)$ is strongly $A(0)$-stable. Then, Theorem 3.1 yields, for sufficiently small $k$ and $k^{-1} h^{2 r}$, the error estimate

$$
\max _{n}\left|u^{n}-U^{n}\right| \leq c\left(k^{p}+h^{r}\right) .
$$

Remark 4.2. By (4.24), we see that (1.4) holds for any $\lambda>0$ with $\mu(v)$ bounded for $v$ bounded in $L^{2(\rho-1)}$. Using this fact, Sobolev's inequality

$$
\|v\|_{L^{s}} \leq C|v|^{1-a}\|v\|^{a}
$$

$a=\nu \frac{s-2}{2 s}, \nu=2,3$, (with $s \leq 6$, of course, for $\nu=3$ ) and Remark 2.1, it is easily seen that the meshcondition $k^{-1} h^{2 r} \leq c$, under which (4.30) holds, can be weakened. We shall not dwell on this.

We close this subsection by briefly considering the case of a general smooth function $f$, as well as the case that $f$ satisfies (4.19) for $\nu=3$ but with $\rho>4$. First, we note that in our analysis it suffices to assume that $B$ is well defined and differentiable on a subspace $\widetilde{V}$ of $V \cap L^{\infty}$ containing $V_{h}$, for all $h$. By tracing back the proof of (4.24) we see that in this case $\mu(v)$ is bounded, provided that

$$
\int_{\Omega}\left|f^{\prime}(v(x))\right|^{2} d x
$$

is bounded. Note that the assumption $k^{-1} h^{2 r} \leq c$, for appropriate $c$, of Theorem 3.1 is only used to show that $\left\|\vartheta^{n}\right\| \leq 1$, which implies (3.19), i.e., that $\mu\left(W^{j}-s \vartheta^{j}\right), s \in(0,1)$, is bounded by a constant. In the case under investigation, by using appropriate inverse inequalities, we show that if stronger meshconditions are satisfied, then $\sup _{0<s<1} \int_{\Omega}\left|f^{\prime}\left(W^{j}-s \vartheta^{j}\right)\right|^{2} d x$ is bounded by a constant independent of $h$ and $k$, and thus $\mu\left(W^{j}-s \vartheta^{j}\right)$ will be bounded by an appropriately defined constant $m$, i.e., (3.19) - and, consequently, the error estimate of Theorem 3.1 - will remain valid. We will distinguish the following cases: $\nu=2$ and general $f, \nu=3$ and $f$ satisfies (4.19) with $\rho>4$, and $\nu=3$ and general $f$.

i. $\nu=2$ and general $f$. First, we note that

$$
\|\chi\|_{L^{\infty}} \leq C|\log (\underline{h})|^{1 / 2}\|\chi\|_{H^{1}} \quad \forall \chi \in V_{h},
$$

with $\underline{h}=\min _{K \in \mathcal{T}_{h}} h_{K}$, cf. [19, p. 67]. Obviously, $\int_{\Omega}\left|f^{\prime}(\chi(x))\right|^{2} d x$ is bounded if $\|\chi\|_{L^{\infty}}$ is bounded. Now,

$$
\max _{0 \leq j \leq n+q-1}\left\|\vartheta^{j}\right\|_{L^{\infty}} \leq C|\log (\underline{h})|^{1 / 2} \max _{0 \leq j \leq n+q-1}\left\|\vartheta^{j}\right\|,
$$

and thus, according to (3.16),

$$
\max _{0 \leq j \leq n+q-1}\left\|\vartheta^{j}\right\|_{L^{\infty}} \leq C_{\star} C|\log (\underline{h})|^{1 / 2}\left(k^{p-1 / 2}+k^{-1 / 2} h^{r}\right) .
$$

Therefore, if $k$ and $h$ are chosen such that $|\log (\underline{h})| k^{2 p-1}$ and $|\log (\underline{h})| k^{-1} h^{2 r}$ are sufficiently small, then $\mu\left(W^{j}-s \vartheta^{j}\right)$ will be bounded, and the convergence results hold.

ii. $\nu=3$ and $f$ satisfies (4.19) with $\rho>4$. If $s \geq 6$, we have

$$
\|\chi\|_{L^{s}(\Omega)} \leq C \underline{h}^{-\frac{s-6}{2 s}}\|\chi\|_{H^{1}(\Omega)} \quad \forall \chi \in V_{h} .
$$


Indeed, employing standard homogeneity arguments, one can show that for an arbritrary element $K \in \mathcal{T}_{h}$,

$$
\|\chi\|_{L^{s}(K)} \leq C h_{K}^{-\frac{s-6}{2 s}}\left(\|\nabla \chi\|_{L^{2}(K)}+\|\chi\|_{L^{6}(K)}\right),
$$

and (4.31) follows in view of (4.20). Hence,

$$
\max _{0 \leq j \leq n+q-1}\left\|\vartheta^{j}\right\|_{L^{2(\rho-1)}} \leq C \underline{h}^{-\frac{\rho-4}{2(\rho-1)}} \max _{0 \leq j \leq n+q-1}\left\|\vartheta^{j}\right\| .
$$

Therefore, if $k$ and $h$ are such that $\underline{h}^{-\frac{\rho-4}{\rho-1}} k^{2 p-1}$ and $k^{-1} \underline{h}^{-\frac{\rho-4}{\rho-1}} h^{2 r}$ are sufficiently small, then, as before,

$$
\max _{0 \leq j \leq n+q-1}\left\|\vartheta^{j}\right\|_{L^{2(\rho-1)}} \leq C_{\star} \underline{h}^{-\frac{\rho-4}{2(\rho-1)}}\left(k^{p-1 / 2}+k^{-1 / 2} h^{r}\right) \leq 1,
$$

$\mu\left(W^{j}-s \vartheta^{j}\right)$ will be bounded in view of (4.24) and (4.26), and our convergence results hold.

iii. $\nu=3$ and general $f$. In this case,

$$
\|\chi\|_{L^{\infty}} \leq C \underline{h}^{-1 / 2}\|\chi\|_{H^{1}} \quad \forall \chi \in V_{h},
$$

as one can see by modifying the proof of the two dimensional case. By the same arguments as for $\nu=2$, the convergence results of this paper hold, provided that $\underline{h}^{-1} k^{2 p-1}$ and $k^{-1} \underline{h}^{-1} h^{2 r}$ are sufficiently small.

\section{REFERENCES}

1. G. Akrivis, High-order finite element methods for the Kuramoto-Sivashinsky equation, RAIRO Modél. Math. Anal. Numér. 30 (1996), 157-183. MR 97e:65095

2. S.M. Allen and J.W. Cahn, A macroscopic theory for antiphase boundary motion and its application to antiphase domain coarsening, Acta Metall. 27 (1979), 1085-1095.

3. M. Crouzeix, Une méthode multipas implicite-explicite pour l'approximation des équations d'évolution paraboliques, Numer. Math. 35 (1980), 257-276. MR 82b:65084

4. M. Crouzeix and P.-A. Raviart, Approximation des équations d'évolution linéares par des méthodes à pas multiples, C.R. Acad. Sc. Paris, Série A 287 (1976), 367-370. MR 54:14377

5. C. W. Cryer, A new class of highly stable methods, BIT 13 (1973), 153-159. MR 48:1469

6. C.M. Elliott and S.-M. Zheng, On the Cahn-Hilliard equation, Arch. Rational Mech. Anal. 96 (1986), 339-357. MR 87k:80007

7. L.C. Evans, H.M. Soner and P.E. Souganidis, Phase transitions and generalized motion by mean curvature, Comm. Pure Appl. Math. 45 (1992), 1097-1123. MR 93g:35064

8. R.D. Grigorieff and J. Schroll, Über $A(\alpha)$-stabile Verfahren hoher Konsistenzordnung, Computing 20 (1978), 343-350. MR 83b:65086

9. E. Hairer and G. Wanner, Solving Ordinary Differential Equations II: Stiff and DifferentialAlgebraic Problems, Springer-Verlag, Berlin Heidelberg, Springer Series in Computational Mathematics v. 14, 1991. MR 92a:65016

10. Y. Kuramoto, Chemical Oscillations, Waves and Turbulence, Springer-Verlag, New York, Series in Synthetics, v. 19, 1984. MR 87e:92054

11. M.N. Le Roux, Semi-discrétisation en temps pour les équations d'évolution paraboliques lorsque l'opérateur dépend du temps, RAIRO 13 (1979), 119-137. MR 80e:65056

12. W.R. McKinney, Optimal error estimates for high order Runge-Kutta methods applied to evolutionary equations, Ph.D. thesis, University of Tennessee, Knoxville, 1989.

13. B. Nicolaenko and B. Scheurer, Remarks on the Kuramoto-Sivashinsky equation, Physica 12 D (1984), 391-395. MR 86d:80007

14. D.T. Papageorgiou, C. Maldarelli and D.S. Rumschitzki, Nonlinear interfacial stability of cone-annular film flow, Phys. Fluids A2 (1990), 340-352. MR 91b:76067

15. G. Savaré, $A(\Theta)$-stable approximations of abstract Cauchy problems, Numer. Math. 65 (1993), 319-336. MR 94h:65062

16. L.L. Schumaker, Spline Functions: Basic Theory, Wiley, New York, 1981. MR 82j:41001 
17. E. Tadmor, The well-posedness of the Kuramoto-Sivashinsky equation, SIAM J. Math. Anal. 17 (1986), 884-893. MR 87g:35117

18. R. Temam, Infinite-Dimensional Dynamical Systems in Mechanics and Physics, SpringerVerlag, New York, 1988. MR 89m:58056

19. V. Thomée, Galerkin finite element methods for parabolic problems, Springer-Verlag, Lecture Notes in Mathematics v. 1054, 1984. MR 86k:65006

20. M. Zlámal, Finite element multistep discretizations of parabolic boundary value problems, Math. Comp. 29 (1975), 350-359. MR 51:7326

21. M. Zlámal, Finite element methods for nonlinear parabolic equations, RAIRO 11 (1977), 93-107. MR 58:19239

Department of Computer Science, University of Ioannina, 45110 Ioannina, Greece

E-mail address: akrivis@cs.uoi.gr

IRMaR, Université de Rennes I, Campus de Beaulieu, F-35042 Rennes, France

E-mail address: michel.crouzeix@univ-rennes1.fr

Department of Mathematics, University of Crete, 71409 Heraklion, Crete, Greece, and iacm, Foundation for Research and Technology - Hellas, 71110 Heraklion, Crete, GREECE

E-mail address: makr@sargos.math.uch.gr 